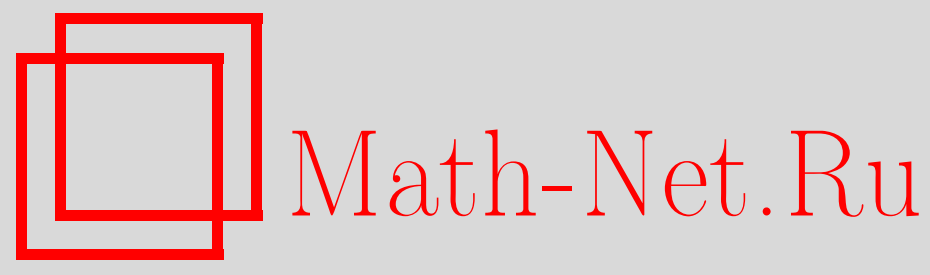

Д. В. Горбачев, Экстремальная задача для периодических функций с носителем в шаре, Матем. заметки, 2001, том 69, выпуск 3, 346-352

DOI: https://doi.org/10.4213/mzm508

Использование Общероссийского математического портала Math-Net.Ru подразумевает, что вы прочитали и согласны с пользовательским соглашением http://www.mathnet.ru/rus/agreement

Параметры загрузки:

IP : 54.157 .27 .8

26 апреля 2023 г., $17: 06: 54$

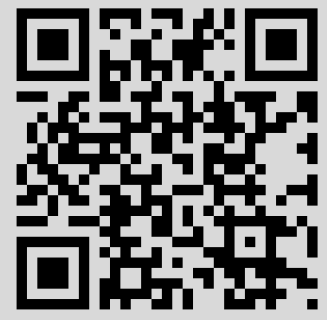




\section{ЭКСТРЕМАЛЬНАЯ ЗАДАЧА ДЛЯ ПЕРИОДИЧЕСКИХ ФУНКЦИЙ С НОСИТЕЛЕМ В ШАРЕ}

\section{Д. В. Горбачев}

Рассмотрена $n$-мерная экстремальная задача П. Турана. В ней требуется найти величину $A_{n}\left(h B^{n}\right)$, равную максимальному нулевому коэффициенту Фурье $\widehat{f_{0}}$ для периодических функций $f$ с носителем в евклидовом шаре $h B^{n}$ радиуса $h$ и неотрицательными коэффициентами Фурье, $f(0)=1$. Данная задача возник ла в связи с приложениями к теории чисел. Величина $A_{1}([-h, h])$ исследовалась С. Б. Стечкиным. Для величины $A_{n}\left(h B^{n}\right)$ получено асимптотическое разложение при $h \rightarrow 0$. Главный член этого разложения определяется из решения $n$-мерной экстремальной задачи для целых функций экспоненциального типа.

Библиография: 7 названий.

Пусть $n \in \mathbb{N}, \mathbb{T}^{n}=[-1 / 2,1 / 2)^{n}-n$-мерный тор, $D \subset[-1,1]^{n}$ - центрально-симметричное тело, $0<h \leqslant 1 / 2, h D \subset \mathbb{T}^{n}$ - гомотет $D ; x y$-скалярное произведение векторов $x, y \in \mathbb{R}^{n},|x|=\sqrt{x x} ; e(t)=\exp (2 \pi i t), \widehat{f}(x)=\int_{\mathbb{R}^{n}} f(y) e(-x y) d y-$ преобразование Фурье функции $f,(f * g)(x)=\int_{\mathbb{R}^{n}} f(x-y) g(y) d y-$ свертка функций $f$ и $g ; \chi_{E}$-характеристическая функция множества $E, \operatorname{vol}(E)$ - объем $E$.

Пусть $K^{n}(h D)$ - класс непрерывных четных функций $f: \mathbb{T}^{n} \rightarrow \mathbb{R}$, обладающих свойствами:

1) $f(x)=\sum_{\nu \in \mathbb{Z}^{n}} \widehat{f}_{\nu} e(\nu x)$, где $\widehat{f}_{\nu}=\int_{\mathbb{T}^{n}} f(x) e(-\nu x) d x-$ коэффициенты Фурье функщии $f$;

2) $\widehat{f}_{\nu} \geqslant 0 \forall \nu \in \mathbb{Z}^{n}$

3) $f(0)=\sum_{\nu \in \mathbb{Z}^{n}} \widehat{f}_{\nu}=1$;

4) $\operatorname{supp} f \subset h D$.

Рассмотрим величину

$$
A_{n}(h D)=\sup _{f \in K^{n}(h D)} \widehat{f_{0}}
$$

П. Тураном в связи с приложениями к теории чисел была поставлена задача оценки $A_{1}(h)=A_{1}([-h, h])$. С. Б. Стечкин показал [1], что $A_{1}(1 / N)=1 / N, N=2,3, \ldots$, откуда

$$
A_{1}(h)=h+O\left(h^{2}\right), \quad h \rightarrow 0 .
$$

Работа выполнена при финансовой поддержке Российского фонда фундаментальных исследований, грант № 00-01-00644, и Минобразования, грант № 97-0-1.4-78. 
Асимптотически экстремальной функцией является периодизация функции

$$
\varphi_{h}(x)=\max \left(1-\frac{|x|}{h}, 0\right)=\left(\chi_{[-1 / 2,1 / 2]} * \chi_{[-1 / 2,1 / 2]}\right)\left(h^{-1} x\right) .
$$

Многомерными вариантами задачи Турана занимался Н. Н. Андреев [2]. Он установил, что для куба $D=[-1,1]^{n}$

$$
A_{n}\left([-h, h]^{n}\right)=h^{n}+O\left(h^{n+1}\right), \quad h \rightarrow 0,
$$

и ромба $D=O^{2}=\left\{x \in \mathbb{R}^{2}:\left|x_{1}\right|+\left|x_{2}\right| \leqslant 1\right\}$

$$
A_{2}\left(h O^{2}\right)=\frac{h^{2}}{2}+O\left(h^{3}\right), \quad h \rightarrow 0
$$

Асимптотически экстремальњыми функциями в обоих случаях являются периодизации сверток

$$
\frac{\left(\chi_{\frac{1}{2} D} * \chi_{\frac{1}{2} D}\right)\left(h^{-1} x\right)}{\operatorname{vol}\left(\frac{1}{2} D\right)}
$$

которые принадлежат $K^{n}(h D)$ в силу свойств свертки [3]: $\operatorname{supp} \chi_{E} * \chi_{E}=2 E, E-$ центрально-симметричное тело, и $\chi_{E} \widehat{*} \chi_{E}=\widehat{\chi}_{E}^{2}$.

В данной работе рассматривается случай евклидова шара $B^{n}=\left\{x \in \mathbb{R}^{n}:|x| \leqslant 1\right\}$, $\operatorname{vol}\left(B^{n}\right)=\pi^{n / 2} / \Gamma(n / 2+1)-$ его объем [3].

TEOPEMA. Для шара $D=B^{n}$

$$
A_{n}\left(h B^{n}\right)=\frac{\pi^{n / 2}}{2^{n} \Gamma(n / 2+1)} h^{n}+O\left(h^{n+2}\right), \quad h \rightarrow 0 .
$$

Асимптотически әкстремальной функиией является периодизация свертки

$$
\frac{\left(\chi_{\frac{1}{2} B^{n}} * \chi_{\frac{1}{2} B^{n}}\right)\left(h^{-1} x\right)}{\operatorname{vol}\left(\frac{1}{2} B^{n}\right)} .
$$

Отметим, что во всех известных случаях главньй член асимптотики $A_{n}(h D)$ равен $\operatorname{vol}\left(\frac{1}{2} D\right) h^{n}$. Нахождение этого члена сводится к решению многомерной экстремальной задачи для целых функций экспоненциального типа. Сформулируем эту задачу.

Пусть $G^{n}(D)$ - класс непрерывных четных функций $g: \mathbb{R}^{n} \rightarrow \mathbb{R}$, удовлетворяющих условиям:

1) $g(y)=\int_{D} \widehat{g}(x) e(y x) d x$

2) $g(y) \geqslant 0 \quad \forall y \in \mathbb{R}^{n}$;

3) $\widehat{g}(0)=\int_{\mathbb{R}^{n}} g(y) d y=1$. 
Найти величину

$$
M_{n}(D)=\sup _{g \in G^{n}(D)} g(0)
$$

Из представления 1) вытекает, что $g$ продолжается до целой функции экспоненциального $2 \pi D^{*}$-типа [3], где $D^{*}$ - поляра множества $D$. Из теоремы о компактности [4, c. 126] следует, что верхняя грань в (3) достигается на некоторой экстремальной функции, которую обозначим $g_{D}$, т.е. $g_{D} \in G^{n}(D)$ и $g_{D}(0)=M_{n}(D)$.

Легко видеть, что функция

$$
g(y)=\frac{\widehat{\chi}_{\frac{1}{2} D}(y)^{2}}{\operatorname{vol}\left(\frac{1}{2} D\right)}=\frac{1}{\operatorname{vol}\left(\frac{1}{2} D\right)} \int_{D}\left(\chi_{\frac{1}{2} D} * \chi_{\frac{1}{2} D}\right)(x) e(y x) d x
$$

принадлежит классу $G^{n}(D)$. Для нее $g(0)=\operatorname{vol}\left(\frac{1}{2} D\right)$, поэтому для величины $M_{n}(D)$ справедлива оценка снизу

$$
M_{n}(D) \geqslant \operatorname{vol}\left(\frac{1}{2} D\right)
$$

Для куба, ромба и шара эта оценка оказывается правильной.

Справедливы следующие две леммы, из которых непосредственно вытекает основная теорема.

ЛЕмма 1. Для произвольных $n$ и $D$

$$
A_{n}(h D)=M_{n}(D) h^{n}+O\left(h^{n+2}\right), \quad h \rightarrow 0 .
$$

Асимптотически әкстремальной является периодизачия функиии $\widehat{g}_{D}\left(h^{-1} x\right)$.

Пусть $j_{\alpha}(t)=2^{\alpha} \Gamma(\alpha+1) t^{-\alpha} J_{\alpha}(t)$ - нормированная функция Бесселя [5, с. 11].

ЛЕмма 2. Для шара $B^{n}$

$$
M_{n}\left(B^{n}\right)=\operatorname{vol}\left(\frac{1}{2} B^{n}\right)=\frac{\pi^{n / 2}}{2^{n} \Gamma(n / 2+1)} .
$$

Экстремальная функиия равна

$$
g_{B^{n}}(y)=\frac{\widehat{\chi}_{\frac{1}{2} B^{n}}(y)^{2}}{\operatorname{vol}\left(\frac{1}{2} B^{n}\right)}=\operatorname{vol}\left(\frac{1}{2} B^{n}\right) j_{n / 2}(\pi|y|)^{2}
$$

Отметим, что из результата Н. Н. Андреева $(2)$ вытекает, что $M_{n}\left([-1,1]^{n}\right)=1$. Следовательно, по лемме 1

$$
A_{n}\left([-h, h]^{n}\right)=h^{n}+O\left(h^{n+2}\right), \quad h \rightarrow 0
$$

что несколько уточняет результаты С. Б. Стечкина и Н. Н. Андреева. 
ДокаЗАТЕЛЬСтво ЛЕммЫ 1. Пусть $g \in G^{n}(D)$ и $g_{h}(y)=h^{n} g(h y)$. Тогда $\widehat{g}_{h}(x)=$ $\widehat{g}\left(h^{-1} x\right)$ [3], supp $g_{h} \subset h D$ и $\widehat{\widehat{g}}_{h}=g_{h}$. Отсюда следует, что периодизация $\widehat{g}_{h}$ равна

$$
f(x)=\sum_{\nu \in \mathbb{Z}^{n}} \widehat{g}_{h}(x+\nu)=\sum_{\nu \in \mathbb{Z}^{n}} g_{h}(\nu) e(\nu x)
$$

и имеет свойства: $f: \mathbb{T}^{n} \rightarrow \mathbb{R}$ - непрерывная четная функция, supp $f \subset h D, \widehat{f}_{\nu}=$ $g_{h}(\nu)=h^{n} g(h \nu) \geqslant 0 \forall \nu \in \mathbb{Z}^{n}, f(0)=\widehat{g}_{h}(0)=\widehat{g}(0)=1$. Следовательно, $f \in K^{n}(h D)$. Так как $\widehat{f_{0}}=h^{n} g(0)$, то получаем оценку снизу

$$
A_{n}(h D) \geqslant \sup _{g \in G^{n}(D)} h^{n} g(0)=M_{n}(D) h^{n} .
$$

Для оценки сверху $A_{n}(h D)$ выберем произвольную непрерывную четную функцию $\varphi: \mathbb{R}^{n} \rightarrow \mathbb{R}$, удовлетворяющую условиям:

1) $\operatorname{supp} \varphi \subset[-1 / 2,1 / 2]^{n}$;

2) $\widehat{\varphi}(y) \geqslant 0 \forall y \in \mathbb{R}^{n}$;

3) $\varphi(0)=\int_{\mathbb{R}^{n}} \hat{\varphi}(y) d y=1$.

Пусть $f$ - произвольная функция из $K^{n}(h D)$. Положим

$$
g_{h}(y)=\int_{h D} f(x) \varphi(x) e(y x) d x
$$

и покажем, что функция $g(y)=h^{-n} g_{h}\left(h^{-1} y\right)$ принадлежит классу $G^{n}(D)$.

Ясно, что $g$ - непрерывная четная функция и $\widehat{g}(x)=\widehat{g}_{h}(h x)=f(h x) \varphi(h x)$. Отсюда, из $(7)$ и равенства $\widehat{g}(0)=f(0) \varphi(0)=1$ вытекает, что она удовлетворяет условиям 1$)$ и 3$)$ класса $G^{n}(D)$. Осталось проверить условие 2$)$.

Так как $h D \subset \mathbb{T}^{n}, \operatorname{supp} f \subset h D, \operatorname{supp} \varphi \subset \mathbb{T}^{n}$, воспользовавшись разложением функции $f$ в ряд Фурье, получим

$$
g_{h}(y)=\int_{\mathbb{T}^{n}} f(x) \varphi(x) e(y x) d x=\sum_{\nu \in \mathbb{Z}^{n}} \widehat{f}_{\nu} \int_{\mathbb{T}^{n}} \varphi(x) e((\nu+y) x) d x=\sum_{\nu \in \mathbb{Z}^{n}} \widehat{f}_{\nu} \widehat{\varphi}(-\nu-y) .
$$

Но $\widehat{f}_{\nu} \geqslant 0$ и $\widehat{\varphi} \geqslant 0 ;$ следовательно, и $g_{h}(y)=h^{n} g(h y) \geqslant 0 \forall y \in \mathbb{R}^{n}$. Условие 2) для функции $g$ выполнено. Таким образом, $g \in G^{n}(D)$.

Для функции $g$ имеем

$$
h^{n} g(0)=g_{h}(0)=\int_{h D} f(x) \varphi(x) d x=\int_{h D} f(x) d x+\int_{h D} f(x)(\varphi(x)-1) d x .
$$

Отсюда

$$
\widehat{f}_{0}=\int_{h D} f(x) d x=h^{n} g(0)+\int_{h D} f(x)(1-\varphi(x)) d x .
$$

Очевидно, что

$$
|f(x)| \leqslant \sum_{\nu \in \mathbb{Z}^{n}} \widehat{f}_{\nu}=1 \quad \forall x \in \mathbb{T}^{n}
$$


и

$$
|\varphi(x)| \leqslant \int_{\mathbb{R}^{n}} \widehat{\varphi}(y) d y=1 \quad \forall x \in \mathbb{R}^{n} .
$$

Следовательно, второе слагаемое в (8) можно оценить так

$$
\left|\int_{h D} f(x)(1-\varphi(x)) d x\right| \leqslant \int_{h D}(1-\varphi(x)) d x .
$$

Отсюда и из (8) имеем оценку $A_{n}(h D)$ сверху

$$
\begin{aligned}
A_{n}(h D) & =\sup _{f \in K^{n}(h D)} \widehat{f_{0}} \leqslant \sup _{g \in G^{n}(D)}\left(h^{n} g(0)+\int_{h D}(1-\varphi(x)) d x\right) \\
& =M_{n}(D) h^{n}+\int_{h D}(1-\varphi(x)) d x .
\end{aligned}
$$

Возникает интересная задача нахождения

$$
\inf _{\varphi} \int_{h D}(1-\varphi(x)) d x .
$$

Можно показать, что эта величина есть $O\left(h^{n+2}\right), h \rightarrow 0$. Это доказьвать не будем, а приведем только функцию $\varphi$, дающую $O\left(h^{n+2}\right): \varphi(x)=\prod_{k=1}^{n} d\left(x_{k}\right)$, где

$$
d(t)= \begin{cases}1-24|t|^{2}+48|t|^{3}, & |t| \leqslant \frac{1}{4} \\ 2(1-2|t|)^{3}, & \frac{1}{4} \leqslant|t| \leqslant \frac{1}{2} \\ 0, & |t| \geqslant \frac{1}{2} .\end{cases}
$$

Преобразование Фурье функции $d$ равно $96\left(\frac{\sin (\pi s / 4)}{\pi s}\right)^{4}[6$, с. 150], т.е. является известным положительным интегральным ядром Джексона-Валле-Пуссена. Поэтому функция $\varphi$ является допустимой. Для нее при $h<1 / 4$

$$
\begin{aligned}
\int_{h D}(1-\varphi(x)) d x & \leqslant \int_{[-h, h]^{n}}(1-\varphi(x)) d x=(2 h)^{n}-\prod_{k=1}^{n} \int_{-h}^{h} d\left(x_{k}\right) d x_{k} \\
& =(2 h)^{n}\left[1-\left(1-8 h^{2}+12 h^{3}\right)^{n}\right]=O\left(h^{n+2}\right) .
\end{aligned}
$$

Отсюда и из (6), (9) окончательно имеем

$$
A_{n}(h D)=M_{n}(D) h^{n}+O\left(h^{n+2}\right)
$$

Из рассуждений, приведенных в начале доказательства, вытекает, что если $g_{D}$ - экстремальная функция в (3), то периодизация $\widehat{g}_{D}\left(h^{-1} x\right)(5)$ принадлежит классу $K^{n}(h D)$ и является асимптотически экстремальной функцией, поскольку ее нулевой коэффициент Фурье равен $M_{n}(D) h^{n}$. Лемма доказана. 
ДоКАЗАТЕЛЬСТВо ЛЕМмы 2. Оценка снизу величины $M_{n}\left(B^{n}\right)$ вытекает из общей оценки снизу (4), поэтому нетривиальной частью является доказательство оценки сверxy.

Пусть $g$ - произвольная функция из $G^{n}\left(B^{n}\right)$. Рассмотрим ее усреднение

$$
g_{0}(t)=\int_{|\xi|=1} g(t \xi) d \xi, \quad t \in \mathbb{C}
$$

Из принадлежности $g$ классу $G^{n}\left(B^{n}\right)$ вытекают следуюшие свойства функции $g_{0}$ :

1) $g_{0}$ - целая четная функция экспоненциального типа $\leqslant 2 \pi[6$, гл. IV];

2) $g_{0}(t) \geqslant 0 \forall t \in \mathbb{R}$

3) $\int_{0}^{\infty} g_{0}(t) t^{n-1} d t=1$

4) $g_{0}(0)=\omega_{n-1} g(0)$, где $\omega_{n-1}=\int_{|\xi|=1} d \xi=2 \pi^{n / 2} / \Gamma(n / 2)-$ площадь единичной сферы [3].

Свойства 2) и 4) очевидны. Свойство 3) вытекает из формулы интегрирования [3]

$$
\int_{\mathbb{R}^{n}} g(y) d y=\int_{0}^{\infty} \int_{|\xi|=1} g(t \xi) d \xi t^{n-1} d t .
$$

Осталось проверить 1$)$. Из свойства 1$)$ класса $G^{n}\left(B^{n}\right)$ следует, что

$$
g_{0}(t)=\int_{|\xi|=1} \int_{B^{n}} \widehat{g}(x) e(t \xi x) d x d \xi=\int_{|x| \leqslant 1} \widehat{g}(x) \int_{|\xi|=1} e(t \xi x) d \xi d x .
$$

Известно [3], что

$$
\int_{|\xi|=1} e(t \xi x) d \xi=\omega_{n-1} j_{n / 2-1}(2 \pi t|x|) .
$$

Отсюда и из (11), если воспользоваться формулой интегрирования (10), получим

$$
\begin{aligned}
g_{0}(t) & =\omega_{n-1} \int_{|x| \leqslant 1} \widehat{g}(x) j_{n / 2-1}(2 \pi t|x|) d x \\
& =\omega_{n-1} \int_{0}^{1} j_{n / 2-1}(2 \pi t s) \int_{|\xi|=1} \widehat{g}(s \xi) d \xi s^{n-1} d s \\
& =\omega_{n-1} \int_{0}^{1} \widehat{g}_{0}(s) j_{n / 2-1}(2 \pi t s) s^{n-1} d s
\end{aligned}
$$

где $\widehat{g}_{0}(s)=\int_{|\xi|=1} \widehat{g}(s \xi) d \xi-$ непрерывная функция. Так как нормированная функция Бесселя $j_{\alpha}$ является целой четной функцией экспоненщиального типа 1 [5], то из (12) следует, что функция $g_{0}$ является целой четной функцией экспоненциального типа $\leqslant 2 \pi$ (см. [6]).

Пусть $0<q_{\alpha+1,1}<q_{\alpha+1,2}<\cdots$ - положительные нули функции Бесселя $J_{\alpha+1}$. Из результатов работы [7] (лемма 4 при $p=0$ и теорема В) вытекает следующее утверждение: для любой целой четной функции $f$ экспоненциального типа $\leqslant 2 \tau$ и

$$
\int_{0}^{\infty}|f(t)| t^{2 \alpha+1} d t<\infty, \quad \alpha>-1
$$


справедлива квадратурная формула

$$
\int_{0}^{\infty} f(t) t^{2 \alpha+1} d t=\frac{2^{2 \alpha+2} \Gamma(\alpha+2)^{2}}{(2 \alpha+2) \tau^{2 \alpha+2}} f(0)+\frac{2}{\tau^{2 \alpha+2}} \sum_{k=1}^{\infty} \frac{q_{\alpha+1, k}^{2 \alpha}}{J_{\alpha+1}^{\prime}\left(q_{\alpha+1, k}\right)^{2}} f\left(\frac{q_{\alpha+1, k}}{\tau}\right) .
$$

Функция $g_{0}$ в силу ее свойств 1$)-3$ ) удовлетворяет условиям этого утверждения при $\alpha=n / 2-1, \tau=\pi$. Поэтому, применяя к функции $g_{0}$ квадратурную формулу и используя ее свойства 2), 4), получим

$$
\begin{aligned}
1 & =\int_{0}^{\infty} g_{0}(t) t^{n-1} d t=\frac{2^{n} \Gamma(n / 2+1)^{2}}{n \pi^{n}} g_{0}(0)+\frac{2}{\pi^{n}} \sum_{k=1}^{\infty} \frac{q_{n / 2, k}^{n-2}}{J_{n / 2}^{\prime}\left(q_{n / 2, k}\right)^{2}} g_{0}\left(\frac{q_{n / 2, k}}{\pi}\right) \\
& \geqslant \frac{2^{n-1} \Gamma(n / 2) \Gamma(n / 2+1)}{\pi^{n}} g_{0}(0)=\frac{2^{n-1} \Gamma(n / 2) \Gamma(n / 2+1) 2 \pi^{n / 2}}{\pi^{n} \Gamma(n / 2)} g(0)=\frac{g(0)}{\operatorname{vol}\left(\frac{1}{2} B^{n}\right)},
\end{aligned}
$$

откуда и следует оценка сверху $g(0) \leqslant \operatorname{vol}\left(\frac{1}{2} B^{n}\right) \forall g \in G^{n}\left(B^{n}\right)$ и $M_{n}\left(B^{n}\right) \leqslant \operatorname{vol}\left(\frac{1}{2} B^{n}\right)$.

Таким образом, $M_{n}\left(B^{n}\right)=\operatorname{vol}\left(\frac{1}{2} B^{n}\right)$ и экстремальной функцией $g_{B^{n}}$ является

Поскольку (см. [5, с. 55] и (11))

$$
\frac{\widehat{\chi}_{\frac{1}{2} B^{n}}(y)^{2}}{\operatorname{vol}\left(\frac{1}{2} B^{n}\right)}
$$

$$
\begin{aligned}
\widehat{\chi}_{\frac{1}{2} B^{n}}(y) & =\int_{|x| \leqslant 1 / 2} e(y x) d x=\omega_{n-1} \int_{0}^{1 / 2} j_{n / 2-1}(2 \pi|y| s) s^{n-1} d s \\
& =\frac{2 \pi^{n / 2} 2^{n / 2-1} \Gamma(n / 2)}{\Gamma(n / 2)(2 \pi|y|)^{n / 2-1}} \int_{0}^{1 / 2} J_{n / 2-1}(2 \pi|y| s) s^{n / 2} d s \\
& =\left.\frac{1}{(2 \pi)^{n / 2}|y|^{n}}(2 \pi|y| s)^{n / 2} J_{n / 2}(2 \pi|y| s)\right|_{0} ^{1 / 2} \\
& =\frac{\pi^{n / 2}}{2^{n} \Gamma(n / 2+1)} j_{n / 2}(\pi|y|)=\operatorname{vol}\left(\frac{1}{2} B^{n}\right) j_{n / 2}(\pi|y|),
\end{aligned}
$$

TO

$$
g_{B^{n}}(y)=\operatorname{vol}\left(\frac{1}{2} B^{n}\right) j_{n / 2}(\pi|y|)^{2} .
$$

Лемма доказана.

\section{СПИСОК ЦИТИРОВАННОЙ ЛИТЕРАТУРЫ}

[1] Стечкин С. Б. Одна экстремальная задача для тригонометрических рядов с неотрицательньми коэффициентами // Избранные труды. Математика. М.: Наука, 1998. С. 244-245.

[2] Андреев Н.Н. Экстремальные задачи для периодических функций с мальм носителем // Вестн. МГУ. Сер. 1. Матем., мех. 1997. № 1. С. 29-32.

[3] Стейн И., Вейс Г. Введение в гармонический анализ на евклидовых пространствах. М.: Мир, 1974.

[4] Никольский С. М. Приближение функций многих переменных и теоремы вложения. М.: Наука, 1977.

[5] Бейтмен Г., Эрдейн А. Высшие трансцендентные функции. Т. 2. М.: Наука, 1966.

[6] Ахиезер Н. И. Лекции по теории аппроксимации. М.: Наука, 1965.

[7] Ghanem R. B., Frappier C. Explicit quadrature formulae for entire functions of exponential type // J. Approx. Th. 1998. V. 92. P. 267-279. 